\title{
Research on the Mechanical Strength of Emulsified Asphalt-Cement- stabilized Macadam Based on Neural Network Algorithm
}

\author{
Chenhao Guo ${ }^{1, *}$, Xianpeng Cheng ${ }^{2}$ and Xiaoming Zhang ${ }^{3}$ \\ ${ }^{1}$ School of Materials Science and Engineering, Chang' an University, Xi'an 710064, China, ${ }^{2} X i^{\prime} a n$ Municipal Engineer- \\ ing \& Research Institute Co., Ltd, Xi'an 710068, China; ${ }^{3}$ Shanxi Academy of Building Research, Xi'an, 710082, China
}

\begin{abstract}
In this paper, we researched the application of mechanical strength experiment on emulsified asphalt cementstabilized macadam based on neural network algorithm. Based on the analysis of the early diseases of asphalt pavement, which uses semi-rigid base, solving the problem of semi-rigid base asphalt pavement's reflection crack, this paper proposes the research approach that uses a modified semi-rigid base, appropriate pavement structure as the point of starting. Combining with the past emulsified asphalt-cement-stabilized macadam material mixing methods, the construction technologies of cement-stabilized macadam and synchronous chip sealing, this paper has come up with a new method for modification of semi-rigid base. The unconfined compressive strength and splitting strength of the mixtures would decrease as the mixing amount of emulsified asphalt increases, and the reduction rate of compressive strength decreases as the curing age grows. The mixing amount of emulsified asphalt has almost no influence on the flexural-tensile strength at normal temperature, so it can be ignored. The experimental result shows that the emulsified asphalt cement-stabilized macadam has good performance than traditional methods.
\end{abstract}

Keywords: Emulsified asphalt-cement-stabilized macadam, mechanical strength, neural network algorithm, simulation.

\section{INTRODUCTION}

With the social progress and constant development of science and technology, there is no denying that road transportation plays a more and more important role in national economy. The more important role the road transportation has, the greater the demand for road quality. In the field of civil engineering, natural building materials have been difficult to meet the requests of the road transportation, which is constantly being developed. Nobody could have failed to notice the fact that new materials and modified materials research are prevalent and pervasive issues with which we are all confronted.

Due to many benefits of cement-stabilized gavel, good intensity, the rigidity and integrity, good water stability and anti-frozen, wide source, it is widely used in the base. But for the understanding of cement-stabilized gravel, base material intensity standard still has some weakness. Bottom tensile stress as the standard is stipulated in Specifications for Design of Highway Asphalt Pavement (JTG DSO-2006), Compressive strength is used as the standard in material composition design and engineering quality. But unconfined compressive strength tested in laboratory nearly has no relation with tensile stress of semi-rigid base's bottom [1]. Based on theoretical research and systematically laboratory tests of cement-stabilized gravel, the proper strength controlling standards and specification were put forward, and this made the standards in design phase that can also be used in the construction phase.

*Address correspondence to this author at the School of Materials Science and Engineering, Chang' an University, Xi'an 710064, China;

Tel: +86 15029229996; E-mail: guochenhao@sohu.com
Mixing emulsified asphalt into cement-stabilized macadam can make mixtures that have the characteristics of rigidity and flexibility [2]. It also can reduce drying shrinkage and increase ultimate strain of the mixtures so as to reduce its shrinkage cracking due to change of temperature and humidity as well as resulted in entire pavement cracks. The incorporation of emulsified asphalt would make mixture have a certain temperature sensitivity, and rising experimental temperature would decrease its strength. The unconfined compressive strength and splitting strength of the mixtures would decrease as the mixing amount of emulsified asphalt increases and the reduction rate of compressive strength decreases as the curing age grows. The mixing amount of emulsified asphalt has almost no influence on the flexural tensile strength at normal temperature, so it can he ignored. The emulsified asphalt cement-stabilized macadam has good resistance to water damage, compressive strength after immersion has no variation and the loss of strength under drying curing condition is less.

\section{THE FRAMEWORK AND NEURAL NET- WORK ALGORITHM}

After adding the emulsified asphalt to the fly-ash cementstabilized soil and the cement-stabilized soil, their unconfined compressive strength and indirect tensile strength has reduced, but it still meets the regulatory requirements. When the emulsified asphalt is between $2 \%$ and $4 \%$, the emulsified asphalt-cement powder-fly-ash stabilized powder meets the technical and regulatory requirements of highways and firstlevel roads base. The case is the same, when the emulsified asphalt is $2 \%$. Meanwhile, the compressive resilient modulus of both the fly-ash cement-stabilized soil and the cement- 
stabilized soil is smaller than that of the semi-rigid material of the same age period.

The water stability and freezing stability of fly-ash cement-stabilized soil are better than that of cement-stabilized soil by adding emulsified asphalt. When the content is $2 \%$, the freezing resistance and abrasive resistance are the optimum. Emulsified asphalt can reduce the deformation of drying shrinkage and temperature shrinkage effectively. Coefficient of shrinkage of emulsified asphalt treated fly-ash cement-stabilized soil and emulsified asphalt treated cementstabilized soil is less than that of emulsified asphalt treated lime stabilized soil obviously. In the same way, the former two materials have the similar rule between their drying shrinkage and temperature shrinkage.

The economic analysis indicates that the semi-rigid base mixed with emulsified asphalt has important social and economic benefits. Especially in the areas which lack highquality granules, it is very economic and reasonable to make use of the semi-rigid base mixed with emulsified asphalt. So it may popularize.

Reflective cracking of asphalt pavement based on semirigid type base is widely existed in our country's highway and becomes one of the difficult problems to deal with. Cracking in base materials (normally cement-stabilized stone) has been shown to be a major source of this distress in roadways. Precise simulation of initiation and propagation of cracking in cement stabilization stone under loading, can avoid time-consuming and expensive indoors experiments. Only by input loads, geometry and material parameters, the Indirect Tensile Test can be accurately simulated, and then useful mechanical parameters can be provided for preventing reflection cracking in the pavement design. The cementstabilized stone is a composite material comprised of cement and stone. The cracking on macro scale is a coalescence of cracking on micro scale, whereas micro-cracking is on the cement and aggregate particulate level. That is why how to link the micro mechanical behavior with macro mechanical behavior is the primary objective of the research work.

A neural network for handwriting recognition is defined by a set of input neurons which may be activated by the pixels of an input image. After being weighted and transformed by a function (determined by the network's designer), the activations of these neurons are then passed on to other neurons. This process is repeated until finally, an output neuron is activated. This determines which character was read.

Like other machine learning methods, systems that learn from data-neural networks have been used to solve a wide variety of tasks that are difficult to solve by using ordinary rule-based programming, including computer vision and speech recognition. Computational devices have been created in CMOS, for both biophysical simulation and neuromorphic computing. More recent efforts show promise for creating Nano-devices [3] for very large scale principal components analyses and convolution. If successful, these efforts could usher in a new era of neural computing [4] that is a step beyond digital computing, because it depends on learning rather than programming, and because it is fundamentally analogous rather than digital even though the first instantiations may in fact be with CMOS digital devices.
Genetic algorithm is a method of forming the research study established by John Holland and his colleagues at the University of Michgan American student in the late 1960s and early 1970 s $[5,6]$. In this method, simulation mechanism of biological evolution Model to construct artificial system, has been widely used in recent years [7]. From Traditional BP neural network algorithm's slow convergence and easy to fall into Local minima shortcomings, this paper is based on genetic algorithm BP Network mechanisms to improve the convergence speed of the network, and then the improved BP Neural networks is used for the evaluation of the level of the university library information.

Each index can score from reviewer's subjective scoring method after obtaining. The data to be used in equation (1) is normalized.

$\bar{x}_{i}=\frac{x_{i}-b_{i}}{a_{i}-b_{i}}$

Where $\mathrm{Xi}$ and $\mathrm{Xj}$ respectively, are the $\mathrm{i}$-th index and the actual value Standard value; ai, bi are the maximum, minimum, of the i-th index. Known evaluation indexes $\mathrm{m}$, $\mathrm{n}$ are the hidden layer nodes depending on problems and experimental data to determine, you can also experience the value of the formula (2) for the decision.

$n=\log _{2} m$

Hidden node output is calculated as follows:

$h_{j}=f\left(\sum_{i=1}^{m} w_{i j} x_{i}-\theta_{j}\right)$

Where $\theta_{\mathrm{j}}$ is defined as the threshold value of hidden node.

The output of the output node is calculated as follows:

$f\left(\sum_{i=1}^{m} w_{i j} x_{i}-\theta_{j}\right)=f\left(f\left(\theta_{j}\right)\right)$

Where in $\theta$ is an output node threshold.

Equation (3) and Equation (4) in the transfer function is generally expressed as $(0,1)$ interval of S-type function:

$f\left(\sum_{i=1}^{m} w_{i j} x_{i}-\theta_{j}\right)=f\left(f\left(\theta_{j}\right)\right)$

Genetic algorithm to train the neural network can be used in the mechanical strength experiment on emulsified asphalt cement-stabilized macadam. The input layer connection weights can be denoted as $\mathrm{w}_{\mathrm{ij}}$, hidden layer and output layer connection weights can be denoted as $t_{j}$, hidden layer threshold can be denoted as $\theta_{\mathrm{j}}$ and the output layer threshold can be denoted as $\theta$. So connected together to form into a long string, the string corresponding to each position of a group of network weights and threshold value, constitutes an individual. It can generate an initial population of $\mathrm{N}$ individuals [8].

Guiding the evolutionary process toward the region of space may contain the best individual conduct. Suitable function selection should have a great impact on the training results. This paper uses a calculation error of the neural network (see equation 6), and the fitness function error. Always 
cutting along the neural network output error in fewer search direction, the convergence target is the minimum output error.

$\mathrm{T}_{\text {low }}$ and $\mathrm{T}_{\text {high }}$ are the key parameters to determine whether the parameter is serious load imbalance. If one node's load is higher than $\mathrm{T}_{\text {high }}$, and there is at least one node, whose load is lower than $\mathrm{T}_{\text {low }}$, then the request will be diverted to the lower one. If a node, whose load is two times higher than $\mathrm{T}_{\text {high }}$, request will be diverted to the lowest node in system, whether there has a node whose load is lower than $T_{\text {low }}$ or not. If all the nodes' load are two times higher than $\mathrm{T}_{\text {high, }}$ efficiency will be reduced, so the total number of effective connections in system must be limited, and the value of $\mathrm{T}_{\text {high }}$ and $\mathrm{T}_{\text {low }}$ must be an appropriate set. Supposing the number of nodes is $n$, the largest number of the total effective connections is L, we can get:

$$
\tau_{i j}(t+n)=\rho \tau_{i j}(t)+\Delta \tau_{i j}
$$

where $\rho$ evaporation is a coefficient such that $(1-\rho)$ represents the of trail between time $t$ and $t+n$

$$
\Delta \tau_{i j}=\sum_{k=1}^{m} \Delta \tau_{i j}{ }^{k}
$$

Then we have:

$$
L=(N-1) * T_{\text {high }}+T_{\text {low }}-1
$$

Thus there always has a node, whose load is lower than $\mathrm{T}_{\text {low. }}$. In order to improve the throughput of system, $\mathrm{T}_{\text {low }}$ should be high enough. If the value of $T_{\text {low }}$ is $T_{\text {high }}$ minus, $\mathrm{T}_{\text {low }}$ is very important to obtain, so the value of $\mathrm{T}_{\text {high }}$ should be set as high as possible. Assuming the $\mathrm{T}_{\text {low }}$ is known, the maximum of request delay minus the minimum of request delay is $\mathrm{D}$, the average request delay is $\mathrm{R}$, then we can get:

$T_{\text {high }}=\frac{\left(T_{\text {low }}+D / R\right)}{2}$

\section{THE EXPERIMENT ON MECHANICAL STRENGTH OF EMULSIFIED ASPHALT- CEMENT-STABILIZED MACADAM BASED ON NNA}

Aggregate grade not only affects strength, stability and construction properties (separation of mixtures, etc.) of cement-stabilized materials, but also affects dry shrinkage properties. In this experiment, it is the detailed aggregate grades taken for all of cement-stabilized materials into account. In order to analyze and compare, it also includes the low and high limit aggregates of the specifications.

To overcome the shortcomings of CCR, CCRDSS was employed as the base course material in the paper. The principle of the structure is to increase the coarse aggregate content so as to make the mixture form tight locked skeleton in order to reduce the shrinkage coefficient, enlarge tensile strength and improve the anti-cracking performance of the mixture. The void of the mixture is filled with cement and fine aggregate, so the maximum density of the mixture can be obtained and the mixture's durability can be raised. The following sections present the evaluation method of anti- cracking performance of CCR and the research process in laboratory and in field.

Raw materials in the research include as follows.

Cement: 425\# Portland Cement, its main technical properties are listed in Table 1.

Crushed Rock Aggregates: the size of each crushed rock aggregate can be classified into the grades of $20-40 \mathrm{~mm}, 10-$ $20 \mathrm{~mm}$ and $5-10 \mathrm{~mm}$ etc. The test results of aggregate are presented in Table 2.

River Sand: its fineness modulus is equal to 2.5 , so it belongs to medium sand.

Suppose the aggregate is a sphere of the diameter of D and is piled up according to the simple cube, that is to say the sphere of the diameter of $\mathrm{D}$ is put into a cubic container of each side D. Under the condition, the aggregate is accumulated to be in the most looseness. So percentage of void content of the container can be calculated as follows:

$$
V=\frac{1-\frac{\pi}{6} D^{3}}{D^{3}}=47.6 \%
$$

Table 1. Cement properties.

\begin{tabular}{|c|c|}
\hline Index & 425\#Portland cement \\
\hline \hline Initial Setting (h:min) & $2: 51$ \\
\hline Initial Setting (h:min) & $3: 23$ \\
\hline Compression Strength (MPa) & 27.1 \\
\hline Bending Strength (MPa) & 5.8 \\
\hline
\end{tabular}

Table 2. Cement initial test results.

\begin{tabular}{|c|c|}
\hline Apparent Density (g/cm3) & $\mathbf{2 . 7 8}$ \\
\hline \hline Loose Density (g/cm3) & 1.46 \\
\hline Percentage of Void (\%) & 45.2 \\
\hline Ratio of Water Absorption (\%) & 0.13 \\
\hline $\begin{array}{c}\text { Aggregate Crushing Value (\%) } \\
\text { Content of Elongated } \\
\text { Aggregate (\%) }\end{array}$ & 15.22 \\
\hline
\end{tabular}

If the sphere is a diameter of $\mathrm{D} / 2$, the container can hold 8 spheroids; If the spheroid is a diameter of $\mathrm{D} / 4$, the container can hold 64 spheroids; the rest may be deduced analogously, but the container's percentage of void content is always not changed, namely $47.6 \%$.

In the so-called Dense Skeleton Structure made of maximum grain-size aggregates $(20-40 \mathrm{~mm}$ crushed rock), are 
used as the main aggregates, whose quantity should not exceeded to that in the naturally loose-laid framework. The quantity of the other grade crushed rock aggregates should be determined by the filling of each grade, which should satisfy the filling of the previous grade void and not interfere with the Skeleton Structure of the previous grade. This kind of Aggregate Gradation with interlocking and filling may be the best mixture in such aspects as friction, cohesive force and compactness. The detailed calculating procedure can be seen as follows.

The apparent density of the main aggregates $(20-40 \mathrm{~mm}$ crushed rock) is usually about $2.68 \mathrm{~g} / \mathrm{cm} 3$, whereas its maximum dry density is commonly $1.92 \mathrm{~g} / \mathrm{cm} 3$. Here, let it take $2.0 \mathrm{~g} / \mathrm{cm} 3$. Because aggregates are not really spheres, it is impossible for the main aggregates to array according to the ideal interlock principle. The percentage of void is usually a little larger than the ideal one, so let it take $50 \%$ when calculating. Therefore, weight percentage of the main aggregates is calculated as follows.

$$
\frac{(1-0.5) \times 2.68}{2.0}=67 \%
$$

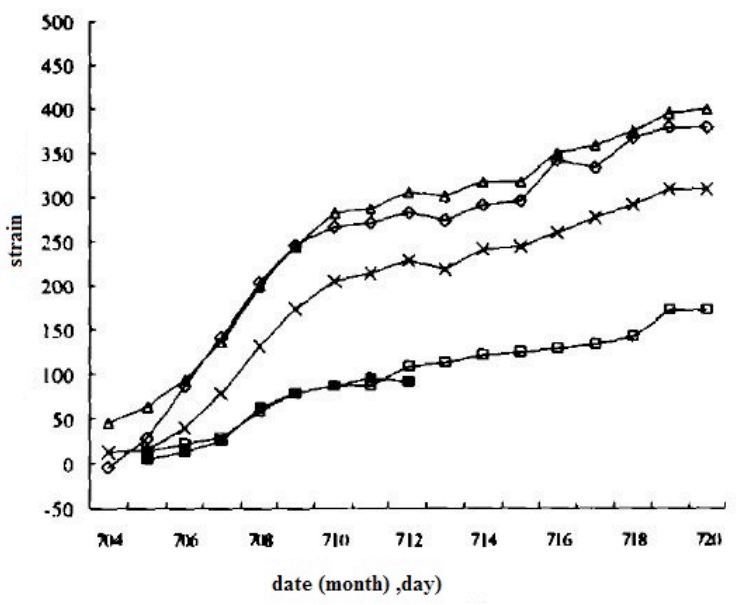

(A1-A5)

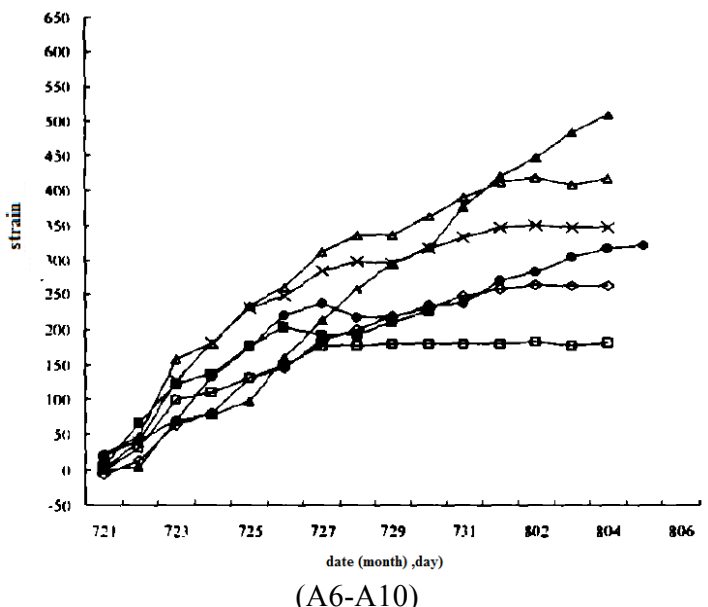

(A6-A10)

Fig. (1). Mechanical strength experiment on strain of small beam with time.
In Fig. (1), "strain 1" means the emulsified asphaltcement-stabilized macadam grade A1, the rest are contrast sets and can be deduced accordingly.

Maximal dry shrinkage strain of mixture A2 is about half that of A11 and A12. Due to containing more coarse aggregates and less fine aggregates, strain of mixture A12 is greatest, which is about 2 times of mixture A11. $26.5 \mathrm{~mm}$ aggregate affects more the dry shrinkage strain of small beam. Dry shrinkage strain of mixture A2 is similar to that of A3. This indicates that, when the total amount of $26.5 \mathrm{~mm}$ aggregate reaches $3 \%$, even its amount is increasing, the effect of ameliorating small beam's shrinkage isn't distinct. By controlling the pass ratio of aggregates of 4.75, 2.36 and $0.075 \mathrm{~mm}$, the effect of ameliorating small beam's shrinkage strain is distinct. The shrinkage strain of mixture A4 or A5 is only $1 / 2$ or $1 / 3$ of mixture A2. And with time going, this difference increases. Thus, during the highway construction, enhancing the control of aggregate grades and making sure the mixture construction continuously and equably are needed. Especially controlling the fine aggregates of 4.75, 2.36 and $0.075 \mathrm{~mm}$ and making sure that aggregate grades lie with the medium value of specifications are the urgent necessities. At the same time, harmonizing the mixing, transportation, spreading and rolling of mixture and enhancing maintenance of cement-stabilized bases are also required.

Optimized by emulsified asphalt-cement-stabilized macadam, fatigue test is set to 500 times the number for tension and compression, with the training Error of 0.001. 10 experts commented on the level of the tension and compression as a test sample. Sample root mean square error of 0.05 , was Library level of tension and compression experiment, and experts predicted values to score more consistent result. Table $\mathbf{3}$ shows the test to validate the model.

Table 3. Test results for fatigue test.

\begin{tabular}{|c|c|c|c|}
\hline $\begin{array}{c}\text { Number of } \\
\text { samples }\end{array}$ & Intact rate & $\begin{array}{c}\text { Predictive } \\
\text { value }\end{array}$ & Error \\
\hline \hline 1 & 0.81 & 0.81003 & 0.0070 \\
\hline 2 & 0.91 & 0.9098 & 0.0191 \\
\hline 3 & 0.64 & 0.6401 & 0.0113 \\
\hline 4 & 0.87 & 0.8707 & 0.0349 \\
\hline 5 & 0.84 & 0.8392 & 0.0367 \\
\hline 6 & 0.79 & 0.8001 & 0.1267 \\
\hline 7 & 0.85 & 0.8493 & 0.0345 \\
\hline 8 & 0.77 & 0.7687 & 0.0447 \\
\hline 9 & 0.73 & 0.731 & 0.0382 \\
\hline 10 & 0.88 & 0.8803 & 0.0230 \\
\hline
\end{tabular}

In order to illustrate the actual effect of using emulsified asphalt-cement-stabilized macadam compared with the traditional cement, we choose different loading nodes to do the test. The comparison before and after using emulsified asphalt-cement-stabilized macadam in fatigue test of tension and compression and can be seen from Fig. (2) and Fig. (3). The result shows that during the same experimental time, the emulsified asphalt-cement-stabilized macadam can achieve better performance in the tension and compression ability than the traditional one. 


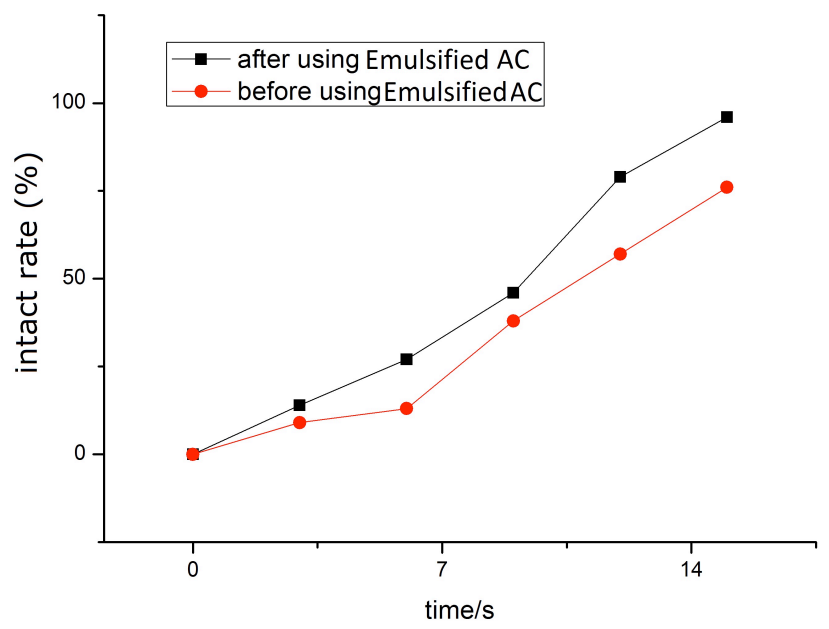

Fig. (2). The comparison before and after using stabilized macadam in fatigue test of tension

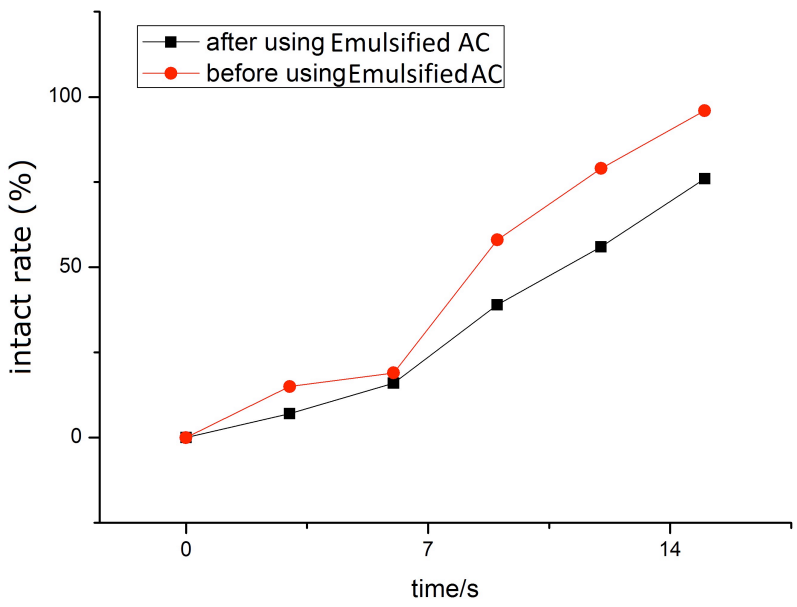

Fig. (3). The comparison before and after using emulsified asphaltcement-stabilized macadam in fatigue test of compressing

\section{CONCLUSION}

In this paper, a new mechanical strength experiment on emulsified asphalt cement-stabilized macadam based on neural network algorithm is presented and researched. Its architecture and features are introduced, and a simulation example of fatigue test of tension and compressing is demonstrated. Laboratory experiments and comparative methods are used to study the mechanical laws of unconfined compressive strength, splitting strength and flexural tensile strength of the mixtures mixed with emulsified asphalt under different curing conditions such as age, temperature etc. The test results show that incorporation of emulsified asphalt would make the mixture have a certain temperature sensitivity, and the rising experimental temperature would decrease its strength. The unconfined compressive strength and splitting strength of the mixtures would decrease as the mixing amount of emulsified asphalt increases, and the reduction rate of compressive strength decreases as the curing age grows. The mixing amount of emulsified asphalt has almost no influence on the flexural tensile strength at normal temperature, so it can be ignored. The emulsified asphalt cement-stabilized macadam has good resistance to water damage, compressive strength after immersion has no variation and the loss of strength under drying curing condition is less.

\section{CONFLICT OF INTEREST}

The authors confirm that this article content has no conflict of interest.

\section{ACKNOWLEDGEMENTS}

This work is supported by the Key Project of Guangxi Social Sciences, China (No. gxsk201424), the Education Science fund of the Education Department of Guangxi, China (No. 2014JGA268), and Guangxi Office for Education Sciences Planning, China (No. 2013C108).

\section{REFERENCES}

[1] J. Tai, W. Meleis, and J. Zhang, Adaptive Resource Allocation for Cloud Computing Environments under Bursty Workloads, Northeastern University, Boston, USA, 2013, pp. 978-987.

[2] J. M. Tirado, D. Higuero, and F. Isaila, "Predictive data grouping and placement ivor cloud-based elastic server infrastructures", $11^{\text {th }}$ IEEE/ACM International Symposium on Cluster, Cloud and grid Computing, IEEE, 2011, pp. 281-294,

[3] Y. Deng, and Z. Du, "The combination of artificial neural network and genetic algorithm applied to forecast of oil and gas yield," Mathematics in Practice and Theory, vol. 38, no.15, pp. 118-123, 2008.

[4] J. Liu, and X. Gan, "Optimum design of self-adaptive wavelet neural networks based on hybrid hierarchy genetic algorithm," Fire Control and Command Control, vol. 33, no. 11, pp. 29-32, 2008.

[5] B. Zhang, and G. Wu, "Cooperation of artificial neural networks and improved genetic algorithms for solving," Computer Engineering and Applications, vol. 45, no. 34, pp.35-37, 2009.

[6] Z. Chen, "Application of genetic algorithm and BP neural network in GDP forecasts," Computer and Digital Engineering, vol.37, no.9, 2009

[7] L.H. Chen, Q.C. Chang, X.G. Chen, and Z.D. Hu, "Using BP neural network to predict the water quality of Yellow River," Journal of Lanzhou University (Natural Sciences), vol. 39, no. 2, pp. 53-56, 2003. (in Chinese)

[8] Z.Y. Guo, Z.Y. Chen, L.Q. Li, B.P. Song, and Y. Lu, "Artificial neural network and its application in regime prediction of groundwater quality," Journal of East China Normal University (Natural Sciences), vol. 1, pp. 84-89, 2001. (in Chinese).

Received: February 06, 2015

Revised: April 18, 2015

Accepted: May 28, 2015

(C) Guo et al.; Licensee Bentham Open.

This is an open access article licensed under the terms of the (https://creativecommons.org/licenses/by/4.0/legalcode), which permits unrestricted, noncommercial use, distribution and reproduction in any medium, provided the work is properly cited. 